EXTENDED REPORT

\title{
Retinal ganglion cells toxicity caused by photosensitising effects of intravitreal indocyanine green with illumination in rat eyes
}

\author{
H K F Yip, T Y Y Lai, K-F So, A K H Kwok
}

Br J Ophthalmol 2006;90:99-102. doi: 10.1136/bjo.2005.076042

See end of article for authors' affiliations .....................

Correspondence to: Dr Alvin K H Kwok, MD, FRCS, Department of Ophthalmology, Hong Kong Sanatorium and Hospital, 2 Village Road, Happy Valley, Hong Kong; alvinkwok@hksh.com

Accepted for publication 1 August 2005

\begin{abstract}
Aim: To investigate the effects of indocyanine green (ICG) with or without illumination on rat retinal ganglion cells (RGC) and retinal morphology.

Methods: Intravitreal injections of $1.0 \mathrm{mg} / \mathrm{ml} \mathrm{ICG} \mathrm{solution} \mathrm{were} \mathrm{performed} \mathrm{in} \mathrm{rat} \mathrm{eyes} \mathrm{with} \mathrm{or} \mathrm{without}$ subsequent illumination for 5 minutes. Eyes in the control group had intravitreal injections of balanced salt solution with illumination. Retrograde labelling of RGC with 6\% Fluoro-Gold was performed 1 month later and RGC densities were compared between the three groups. Light microscopy with measurements of outer nuclear layer (ONL) and inner nuclear layer (INL) thicknesses were also performed and compared. Results: Eyes with ICG without illumination showed insignificant reduction in RGC density compared with the control group $(p=0.28)$, whereas a significant decrease in RGC density was found in eyes that had ICG injection with illumination $(p=0.036)$. A significant increase in ONL thickness was also observed in the ICG with illumination treated eyes compared with the ICG without illumination and the control groups $(p<0.001)$. No significant difference in INL thickness was observed between the three groups.

Conclusions: Intravitreal injection of $0.1 \mathrm{mg} / \mathrm{ml} \mathrm{ICG}$ in rat eyes followed by illumination resulted in photosensitising toxicity to RGC. Lower ICG concentration or illumination level should be considered when performing ICG assisted macular surgery.
\end{abstract}

I ntraoperative application of indocyanine green (ICG) dye for internal limiting membrane (ILM) staining to assist its removal has been shown to improve the anatomical success rate in macular hole surgery. ${ }^{1-5}$ However, the use of ICG staining during macular surgery may result in retinal toxicity as adverse effects like visual field defects and poorer visual have been reported..$^{6-9}$ In order to gain more understanding into the pathogenesis of potential ICG related retinal toxicity, in vitro and in vivo experiments have been performed to determine various mechanisms of retinal toxicity. ${ }^{10-21}$ Using in vitro cell cultures, studies have shown that ICG may result in concentration dependent reduction in the cell viabilities in retinal pigment epithelia (RPE), ${ }^{11-13}$ retinal ganglion cell (RGC), ${ }^{14}$ and glial cells. ${ }^{15}$ In vivo animal experiments have also demonstrated that ICG may cause functional and morphological retinal toxicity as demonstrated by histological examination and electroretinography. ${ }^{14}{ }^{16-21}$

The exact pathogenesis of ICG related retinal toxicity is uncertain. Based on results from multifocal electroretinogram, optical coherence tomography and fluorescein angiography, Horio and Horiguchi suggested that the functional damage might also be related to the effects of ICG staining on ganglion cells and their axons.' One of the proposed mechanism of ICG related retinal toxicity is the photosensitising effects of ICG associated with intraoperative illumination. ${ }^{11}{ }^{15}{ }^{21-24}$ In a previous animal study, it has been shown that ICG with endoillumination caused morphological retinal changes as well as electroretinographic changes in rabbit eyes. ${ }^{21}$ As previous studies in the literature mainly investigated ICG related toxicity on outer retinal structures like RPE cells and photoreceptors, it is unclear whether intraoperative application of ICG with illumination will cause toxicity to RGC as a result of photosensitising effects. In this study, we investigated the potential retinal photosensitising toxicity to RGC and retinal morphological changes in rat eyes after intravitreal injection of ICG with or without illumination through retrograde labelling of RGC and histological examinations.

\section{MATERIALS AND METHODS \\ Animals}

Adult Sprague-Dawley rats (female, $200 \mathrm{~g}, 8$ weeks old; Charles River Laboratories Inc, Wilmington, MA, USA) were obtained from the laboratory animal unit of the University of Hong Kong and used in this study. All experiments were conducted in accordance to the ARVO statements for the use of animals in ophthalmic and vision research. The research protocol of this study was approved by the committee on the use of live animals in teaching and research, the University of Hong Kong.

\section{Preparation of ICG solution}

An amount of $25 \mathrm{mg}$ of commercially available ICG (Diagnogreen, Daiichi Pharmaceutical, Tokyo, Japan) was reconstituted with $5 \mathrm{ml}$ of a balanced salt solution (BSS) provided (BSS Plus, Alcon, Fort Worth, TX) to produce a $0.5 \%$ $(5 \mathrm{mg} / \mathrm{ml})$ solution. The mixture was well shaken for 3 minutes before further dilution with BSS to provide solutions with a final concentration of $1.0 \mathrm{mg} / \mathrm{ml}$. The osmolarities of the diluted ICG solution and BSS solution were $300 \mathrm{mOsmM}$ and $302 \mathrm{mOsM}$ respectively.

\section{Intravitreous injection and illumination}

All animals were anaesthetised with an intraperitoneal injection of a xylazine/ketamine mixture (xylazine $8 \mathrm{mg} / \mathrm{kg}$ and ketamine $80 \mathrm{mg} / \mathrm{kg}$ ). Intravitreal injections was performed in rat eyes using methods as previously described. ${ }^{25}$

Abbreviations: BSS, balanced salt solution; ICG, indocyanine green; ILM internal limiting membrane; INL, inner nuclear layer; $\mathrm{ONL}$, outer nuclear layer; PBS, phosphate buffered saline; RGC, retinal ganglion cells 


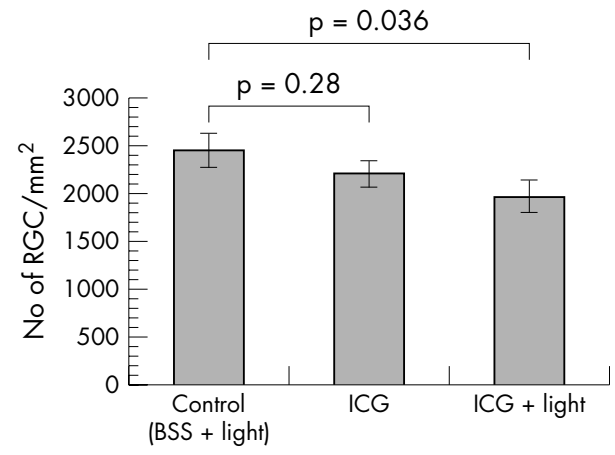

Figure 1 The effects of ICG with endoillumination on rats RGC in vivo. The mean density of RCG was determined with retrograde labelling 1 month after intravitreal injection. The number of Fluoro-Gold labelled RGC decreased significantly in the ICG with illumination group compared with control.

Briefly, pupils were dilated with $0.5 \%$ tropicamide and intravitreal injections were performed by insertion of a 33 gauge needle guided by a stereoscopic microscope. Care was taken to avoid damage to the lens or retina. In order to study the potential toxicity caused by photosensitising effects of ICG, the eyes were divided into three groups: (1) intravitreal BSS injection with illumination (control group); (2) intravitreal ICG injection alone (ICG group); and (3) intravitreal ICG injection with illumination (ICG + light group). A single injection of $8 \mu \mathrm{l}$ of ICG or BSS solution was delivered into the mid-vitreous over 1 minute. After intravitreal injection of the ICG or BSS solutions, all eyes were exposed to light from a standard endoillumination light pipe positioned $5 \mathrm{~mm}$ away from the cornea for 5 minutes. The light source was provided by a standard vitrectomy machine (Millennium, Bausch \& Lomb, Rochester, NY, USA) with an emission spectrum from $420-800 \mathrm{~nm}$ and a luminance of $61.8 \mathrm{~cd} / \mathrm{mm}^{2}$.

\section{Measurement of RGC density}

One month after intravitreal injections, retrograde labelling of RGC was performed using gelfoams soaked with 6\% Fluoro-Gold (Fluorochrome, Denver, CO, USA) applied to the proximal optic nerve stump after a cut was made $0.5 \mathrm{~mm}$ from the eyeball under anaesthesia. Two days later, the rats

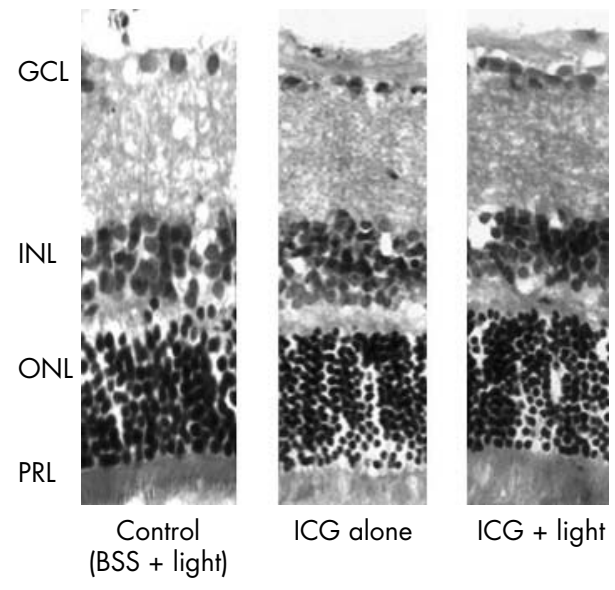

Figure 2 Light microscopy of retinal specimens from the control, ICG alone and ICG plus light groups (haematoxylin and eosin, H\&E stain). All specimens taken from each group showed preservation of the normal retinal layers morphologically. GCL, ganglion cell layer, INL, inner nuclear layer; ONL, outer nuclear layer; PRL, photoreceptor layer. were sacrificed with an overdose of xylazine/ketamine mixture. Animals were first perfused with $0.01 \mathrm{M}$ phosphate buffered saline (PBS) at $\mathrm{pH} 7.4$ and then with a solution containing $4 \%$ paraformaldehyde in $0.01 \mathrm{M}$ PBS. Eyes were enucleated and postfixed in the same solution for 2 hours at $4^{\circ} \mathrm{C}$. Four radial cuts were made in the peripheral retina and the retina was separated and divided into four quadrants. The retinas were flat mounted with the vitreous side facing up on gelatin coated slides in 30\% glycerin. The slides were then examined under ultraviolet illumination on a Zeiss axiophot fluorescent microscope. Labelled RGC were counted along the median line of the four quadrants starting from the optic disc to the peripheral border of the retina at $500 \mu \mathrm{m}$ intervals, under an eyepiece grid of $200 \times 200 \mu \mathrm{m}$. The mean density of labelled RGC of each retina was calculated by the number of labelled RGC in the retina divided by the area of the retina. All measurements were performed by an observer masked to the experimental group.

\section{Light microscopy and ONL and INL thickness measurement}

Sagittal sections were also performed for retinal morphological analysis and measurements of the outer nuclear layer (ONL) and inner nuclear layer (INL) thicknesses. Retinas were cryoprotected in $30 \%$ sucrose and sectioned at $10 \mu \mathrm{m}$ thickness. Sections were stained with haematoxylin and eosin and examined under light microscopy. The thicknesses of the ONL and INL were measured with an image analysis system (Neurolucida, MicroBrightField, Colchester, VT, USA).

\section{Statistical analysis}

Statistical analysis was carried out using a SPSS v1 1.5 (SPSS Inc, Chicago, IL, USA). One way ANOVA with post hoc LSD tests were used to compare the RGC density, and the ONL and INL thickness in the three groups. A p value $<0.05$ was considered statistically significant.

\section{RESULTS}

\section{Retinal ganglion cell density}

Whole mount retina RGC density measurements were performed in retinal specimens from the control, ICG, and the ICG + light groups 1 month and 2 days after intravitreal injections. Fluoro-Gold labelled RGCs were detected in all 21 eyes examined. RGC densities for the control, ICG, and ICG + light groups were 2461 (176) cells $/ \mathrm{mm}^{2}$ (mean (SEM)), 2213 (134) cells $/ \mathrm{mm}^{2}$, and 1979 (166) cells $/ \mathrm{mm}^{2}$, respectively (fig 1). There was a significant reduction in RGC density in the ICG + light group compared with the control group (one way ANOVA post hoc LSD, $p=0.036)$. No significant

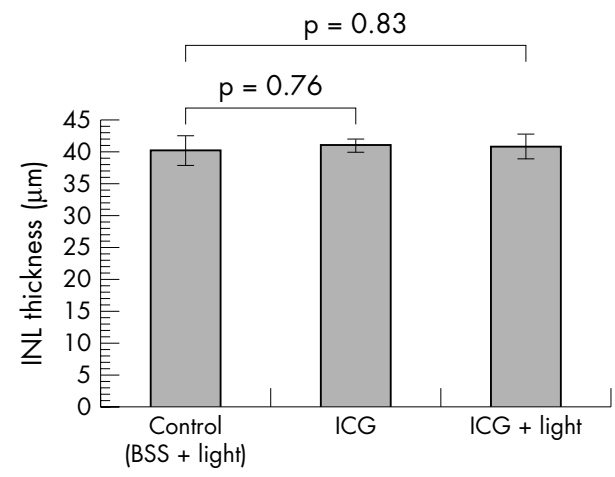

Figure 3 The effects of ICG with illumination on the inner nuclear layer (INL) thickness. No significant difference in the mean INL thickness was found between the three groups. 


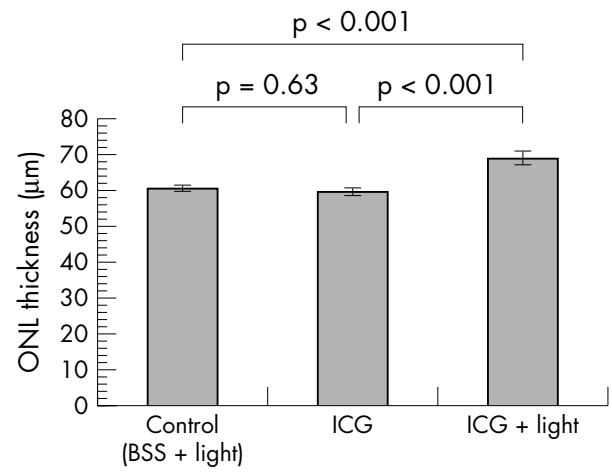

Figure 4 The effects of ICG with endoillumination on the outer nuclear layer (ONL) thickness. A significant increase in the mean ONL thickness was found compared with the control and ICG without illumination groups.

difference in RGC density was found between the control group and the ICG alone group (one way ANOVA post hoc LSD, $\mathrm{p}=0.28$ ).

\section{Retinal morphology and INL and ONL thickness on light microscopy}

One month after intravitreal injections, all the retinal layers appeared normal morphologically in all three treated groups (fig 2). There was no localised destruction in the retinal architecture observed in any of the specimens under light microscopy. Measurements of INL and ONL thickness were performed on the retinal sections in the three groups. The thickness of the INL for the control, ICG and ICG + light groups were $40.2(2.3) \mu \mathrm{m}$ (mean (SEM)), 41.0 (1.0) $\mu \mathrm{m}$, and 40.8 (1.9) $\mu \mathrm{m}$, respectively (fig 3 ). There was no significant difference in the mean INL thickness between the three groups (one way ANOVA, $\mathrm{p}=0.95$ ). The thickness of the ONL for the control, ICG and ICG + light groups were 60.5 (0.9) $\mu \mathrm{m}$ (mean (SEM)), 59.5 (1.1) $\mu \mathrm{m}$, and 68.9 (1.9) $\mu \mathrm{m}$, respectively. There was a significant increase in the ONL thickness for the ICG with endoillumination group compared with both the ICG alone and the control BSS with endoillumination groups (one way ANOVA, $\mathrm{p}<0.001$ ) (fig 4).

\section{DISCUSSION}

In this study, we demonstrated that there was a significant reduction in RGC density in rat eyes following intravitreal injection of $8 \mu \mathrm{l}$ of $1.0 \mathrm{mg} / \mathrm{ml}$ ICG solution with illumination compared with the control group of BSS injection with illumination. Although a small reduction in RGC density was also observed in eyes after $1.0 \mathrm{mg} / \mathrm{ml}$ ICG injection alone, the difference was not significant when compared with the control group. We also observed that there was a significant increase in the ONL thickness in eyes treated with ICG and light compared with the control and the ICG only groups. Our results confirmed the hypothesis suggested in previous in vitro experiments that application of ICG under illumination may result in retinal toxicity as a result of the photosensitising effects of ICG. ${ }^{11} 152324$

Yam et al have previously demonstrated that the application of ICG together with light resulted in a concentration dependent reduction in RPE cell viability and increased expressions of apoptosis related genes p53 and bax as well as the cell cycle arrest protein p2l in human cultured RPE cells. ${ }^{11}$ Such reduction was absent in RPE cells treated with ICG only without illumination. In another study by Jackson et al, application of ICG with illumination resulted in a significant reduction in cell viability in glial cell culture compared with cells treated with ICG without illumination. ${ }^{15}$ Gandorfer et al also demonstrated in human donor eyes that retinal damage may occur after application of ICG with exposure to light of wavelengths longer than $620 \mathrm{~nm}^{22}$ Together with the results obtained from this in vivo study, it is likely that RGC toxicity after intravitreal ICG injection is also a result of the photosensitising effects of ICG.

The reduction in RGC density after intravitreal ICG injection and illumination observed in our study is different from the results obtained by Iriyama et al as they reported that no reduction in viable RGC was observed in vitro after purified RGC were exposed to 1 minute of ICG followed by illumination for 15 minutes. ${ }^{14}$ This difference is likely to be caused by the prolonged contact time of ICG solution in rat eyes in vivo since no attempt was carried out to aspirate the dye from the rat eyes after intravitreal injection. Consistent with our finding, a concentration dependent toxicity to the RGC cell culture is observed after a prolonged 3 days exposure of ICG. $^{14}$ Such concentration dependent RGC toxicity can also be observed in vivo in rat eyes 14 days after intravitreal injection of ICG without illumination. ${ }^{14}$

Besides the photosensitising toxicity of ICG to the RGC, we also observed that there was a significant increase in the ONL thickness following ICG injection with illumination. The ONL is formed by the cell bodies of the photoreceptors and the increase in thickness might be caused by cellular or pericellular oedema. Since we only investigated the effects of ICG on RGC 1 month after injection, we were unable to determine whether such changes are reversible as a result of transient cellular damage or are irreversible. Moreover, the changes in cell densities or cell sizes in the ONL and INL were not assessed in this study and therefore the significance of the increase in the ONL thickness remained uncertain. Previous animal studies have shown that ICG may cause retinal morphological damage resulting in the loss of photoreceptors outer segments. ${ }^{19} 21$ Experiments in rabbit eyes have also demonstrated an increase in apoptosis of photoreceptors and RPE cells 1 day after intravitreal injection of higher concentration of ICG solution without illumination. ${ }^{19}$ Further studies to investigate the potential ICG related photosensitising toxicity to the photoreceptors are warranted.

In conclusion, our study showed that intravitreal injection of $1.0 \mathrm{mg} / \mathrm{ml}$ ICG may result in photosensitising toxicity to RGC in rats with the presence of illumination. Our results should be interpreted with cautions as the vitreous volume around 50-60 $\mu \mathrm{l}$ in rat eyes are much smaller than that of human eyes and higher concentration of ICG will therefore accumulate in rat eyes. Moreover, the dye was injected into the vitreous cavity and was not removed but left for 1 month. This would have resulted in prolonged contact with the retina and increased the potential for toxicity. Another shortcoming of this study was the illumination used was transcorneal rather than within the eye and therefore the standard vitrectomy surgery condition could not be fully simulated. This study also only investigated one concentration of ICG and we were unable to demonstrate the concentration dependent toxicity caused by ICG. None the less, previous studies have already demonstrated that such concentration dependent toxicity occur following intravitreal ICG injection in rat eyes. However, in view of the potential retinal toxicity, clinicians should consider using the minimal concentration of ICG if it is required during surgery. Preparing the ICG solution with sodium free BSS or with dextrose may also help to reduce the retinal toxicity. ${ }^{10}{ }^{24}$ Moreover, surgeons may also consider using lower light levels, shorter light exposure, as well as using different wavelengths of light in order to avoid potential phototoxicity caused by ICG. 


\section{Authors' affiliations}

H K F Yip, K-F So, A K H Kwok, Department of Anatomy, The University of Hong Kong, Pokfulam, Hong Kong SAR, People's Republic of China T Y Y Lai, Department of Ophthalmology and Visual Sciences, The Chinese University of Hong Kong, Hong Kong Eye Hospital, Hong Kong SAR, People's Republic of China

A K H Kwok, Department of Ophthalmology, Hong Kong Sanatorium and Hospital, Hong Kong SAR, People's Republic of China

\section{REFERENCES}

1 Kadonosono K, Itoh N, Uchio $\mathrm{E}$, et al. Staining of internal limiting membrane in macular hole surgery. Arch Ophthalmol 2000;118:1116-18.

2 Da Mata AP, Burk SE, Riemann CD, et al. Indocyanine green-assisted peeling of the retinal internal limiting membrane during vitrectomy surgery for macular hole repair. Ophthalmology 2001;108:1187-92.

3 Kwok AK, Lai TY, Chan WM, et al. Indocyanine green staining and removal of retinal internal limiting membrane in stage 3 or 4 macular hole surgery. Br J Ophthalmol 2003;87:71-4.

4 Kwok AK, Lai TY, Yuen KS, et al. Macular hole surgery with or without indocyanine green stained internal limiting membrane peeling. Clin Experiment Ophthalmol 2003;31:470-5.

5 Slaughter K, Lee IL. Macular hole surgery with and without indocyanine green assistance. Eye 2004;18:376-8.

6 Haritoglou C, Gandorfer A, Gass CA, et al. Indocyanine green-assisted peeling of the internal limiting membrane in macular hole surgery affects visual outcome: a clinicopathologic correlation. Am J Ophthalmol 2002;134:836-41.

7 Ando F, Sasano K, Ohba N, et al. Anatomic and visual outcomes after indocyanine green-assisted peeling of retinal internal limiting membrane in idiopathic macular hole surgery. Am J Ophthalmol 2004;137:609-14.

8 Uemura A, Kanda S, Sakamoto $Y$, et al. Visual field defects after uneventful vitrectomy for epiretinal membrane with indocyanine green-assisted internal limiting membrane peeling. Am J Ophthalmol 2003; 136:252-7.

9 Horio N, Horiguchi M. Effect on visual outcome after macular hole surgery when staining the internal limiting membrane with indocyanine green dye. Arch Ophthalmol 2004;122:992-6.

10 Stalmans $\mathbf{P}$, Van Aken EH, Veckeneer $M$, et al. Toxic effect of indocyanine green on retinal pigment epithelium related to osmotic effects of the solvent. Am J Ophthalmol 2002;134:282-5.
11 Yam HF, Kwok AK, Chan KP, et al. Effect of indocyanine green and illumination on gene expression in human retinal pigment epithelial cells. Invest Ophthalmol Vis Sci 2003;44:370-7

12 Ho JD, Tsai RJ, Chen SN, et al. Cytotoxicity of indocyanine green on retinal pigment epithelium: implications for macular hole surgery. Arch Ophthalmol 2003:121:1423-9.

13 Rezai KA, Farrokh-Siar L, Ernest JT, et al. Indocyanine green induces apoptosis in human retinal pigment epithelial cells. Am J Ophthalmo 2004; 137:931-3.

14 Iriyama A, Uchida S, Yanagi Y, et al. Effects of indocyanine green on retinal ganglion cells. Invest Ophthalmol Vis Sci 2004;45:943-7.

15 Jackson TL, Hillenkamp J, Knight BC, et al. Safety testing of indocyanine green and trypan blue using retinal pigment epithelium and glial cell cultures. Invest Ophthalmol Vis Sci 2004:45:2778-85.

16 Lee JE, Yoon TJ, Oum BS, et al. Toxicity of indocyanine green injected into the subretinal space: subretinal toxicity of indocyanine green. Retina 2003;23:675-81

17 Kawaji T, Hirata A, Inomata Y, et al. Morphological damage in rabbit retina caused by subretinal injection of indocyanine green. Graefes Arch Clin Exp Ophthalmol 2004;242:158-64.

18 Maia M, Kellner L, de Juan E Jr, et al. Effects of indocyanine green injection on the retinal surface and into the subretinal space in rabbits. Retina 2004;24:80-91.

19 Enaida H, Sakamoto T, Hisatomi T, et al. Morphological and functional damage of the retina caused by intravitreous indocyanine green in rat eyes. Graefes Arch Clin Exp Ophthalmol 2002;240:209-13.

20 Maia M, Margalit E, Lakhanpal R, et al. Effects of intravitreal indocyanine green injection in rabbits. Retina 2004;24:69-79.

21 Kwok AK, Lai TY, Yeung CK, et al. The effects of indocyanine green and endoillumination on rabbit retina: an electroretinographic and histologic study. Br J Ophthalmol 2004;88:1590-4.

22 Gandorfer A, Haritoglou C, Gandorfer A, et al. Retinal damage from indocyanine green in experimental macular surgery. Invest Ophthalmol Vis Sci 2003:44:316-23.

23 Haritoglou C, Gandorfer A, Schaumberger M, et al. Light-absorbing properties and osmolarity of indocyanine-green depending on concentration and solvent medium. Invest Ophthalmol Vis Sci 2003;44:2722-9.

24 Ho JD, Chen HC, Chen SN, Tsai RJ. Reduction of indocyanine greenassociated photosensitizing toxicity in retinal pigment epithelium by sodium elimination. Arch Ophthalmol 2004;122:871-8.

25 Mizuno K, Koide T, Yoshimura M, et al. Neuroprotective effect and intraocular penetration of nipradilol, a $\beta$-blocker with nitric oxide donative action. Invest Ophthalmol Vis Sci 2001;42:688-94. 